\title{
Les voies de l'accomplissement au travail en oncologie : facteurs institutionnels, personnels et politiques
}

\author{
Ways to Accomplishment at Work in Oncology: \\ Institutional, Personal and Political Factors
}

\author{
M.-F. Bacqué \\ (C) Lavoisier SAS 2015
}

Pendant des dizaines d'années, l'épuisement professionnel a été conçu comme une souffrance liée à des facteurs personnels. Certes, il était admis que des métiers « à risque » conduisaient de façon préférentielle à la battle fatigue, celle qui consumait les forces du « vaillant petit soldat », mais la métaphore guerrière n'omettait jamais de signaler combien les victimes étaient jeunes, idéalistes et sans ressource. Les professions d'aide étaient au premier plan, mais surtout celles qui approchaient la mort d'autrui, mort réelle dans les services hospitaliers, chez les pompiers ou dans la police, mais aussi mort symbolique comme chez les enseignants dont la responsabilité portait sur toute la vie de leurs élèves. De même que les suicides au travail étaient souvent attribués à un état dépressif sous-jacent, le burn-out ne semblait pas arriver à n'importe quel soignant. Or, les études les plus récentes montrent que l'épuisement professionnel survient dans une institution ou dans un groupe confronté à de lourds enjeux vitaux, lorsque manque la reconnaissance de ce tribut, aussi bien au niveau de la charge de travail que de la distance nécessaire avec elle. Ainsi, parmi les variables les plus fréquentes du burn-out, l'absence de cohérence au travail est la plus douloureusement vécue. Avoir des objectifs, employer ses compétences et sa créativité, coopérer et communiquer avec ses pairs dans une forme de contrat social commun forment la ligne de base du fonctionnement du groupe soignant. Enfin, la convivialité et l'échange, dans la souplesse mentale, permettent aux équipes de renouveler sans cesse leur recherche de qualité et ainsi de contribuer à augmenter leur satisfaction au travail.

La morbidité au travail ne se mesure en effet pas seulement en termes d'absentéisme. Comme nous le montrent les contributeurs de ce numéro, les prémisses du burn-out se manifestent dans la mécanisation des gestes et des mots, dans les consommations de substituts (nourriture, alcool,

M.-F. Bacqué ( $\square)$

Rédactrice en chef de Psycho-Oncologie, EA 3071,

université de Strasbourg, F-67000 France

e-mail : mfbacque@club-internet.fr psychotropes) pour pallier l'épuisement émotionnel et, surtout, dans la perte du sens au travail.

Retrouver l'accomplissement, cette dimension fondamentale issue des travaux de Freudenberger et de Maslach passe par plusieurs voies :

- la voie de l'institution. Elle doit agir par la prévention primaire. Une pédagogie tout d'abord. Celle-ci porterait aussi bien sur les éléments vocationnels (ce qui me conduit à choisir ce métier) que sur les représentations (ce dont je rêve en choisissant ce métier). L'institution soignante est déjà largement reliée à l'université. Elle devrait l'être plus encore afin que les étudiants puissent faire sans cesse appel à leurs formateurs sur le terrain. De même, pour que les savoirs ne se sclérosent pas, il serait utile de revenir régulièrement en formation. Or, si les soignants bénéficient de quelques possibilités de formation continue, les médecins ont accès à de nombreuses nouvelles connaissances sur un mode purement « cognitif». Or, seule la possibilité de mise en pratique permet de vivre émotionnellement les cas difficiles d'annonce, de conflits interpersonnels et de questionnement éthique. Toute la pédagogie doit être révisée et articulée entre médecins et autres soignants. L'utilitarisme a trop longtemps prévalu à l'hôpital pour que l'on puisse parler de formation. La mise à plat des horaires des internes et la reconnaissance de leurs besoins de se reposer sont un premier pas. La PACES et la mise en commun de la première année des études pour médecins et soignants en sont un autre. Les études en sciences infirmières se poursuivant au-delà de la licence pourraient être en France une voie de la reconnaissance des métiers. Enfin, n'oublions jamais que l'hôpital s'ouvre sur le terrain et que des échanges de qualité avec les praticiens libéraux limiteraient aussi bien des causes d'épuisement chez les médecins et infirmiers de ville ;

- la voie personnelle. Elle est encore mal perçue, car nombreux sont ceux qui ne conçoivent pas que les qualités professionnelles soient précédées de qualités personnelles. Cette idée classique ne se contente pas de quelques 
« hochets pédagogiques ». Encore trop souvent, des sessions de formation " clé en main » prétendent apporter des compétences en communication qui pallieront la faiblesse des qualités morales du soignant. Il est rare hélas qu'un tel miracle ait lieu. Tous ces changements sont issus d'un travail au long court. C'est pourquoi penser apprendre à « gérer » ses affects ou ses émotions est un leurre. La dimension économique de cette énergie psychique n'est qu'une partie apparente. La réalité est qu'acquérir une aptitude à avoir conscience de ce que l'on ressent et à contrôler au mieux ses affects pour une utilisation pondérée par la nature d'une situation est un long apprentissage. L'exemple le plus frappant nous est fourni par les évaluations des fluctuations de l'empathie des étudiants en médecine. Cette empathie, énergie psychique nécessaire pour comprendre l'expérience d'autrui, diminue au fur et à mesure des études médicales (toutes les recherches sur ce sujet ne retrouvent pas les mêmes résultats). On imaginerait que le contraire fut nécessaire pour devenir un bon médecin. L'étudiant privilégie en fait, de plus en plus le raisonnement face au patient, au détriment du soutien émotionnel qu'il laisse à d'autres. Le médecin senior établit, quant à lui, des mécanismes de défense face aux émotions, il se place en retrait plus aisément et consacre son énergie et son temps à la direction de son équipe. Cependant, à la faveur d'une identification qui lui échappe, un chef de service peut retrouver toute la puissance d'un investissement affectif comme au premier jour de son exercice. L'empathie n'est donc pas si simple à maitriser. Les psychologues, pour lesquels il s'agit de l'outil princeps, connaissent bien sa variabilité au travers de l'analyse de leur contre-transfert (éprouvé subjectif projeté sur un patient et rappelant des investissements affectifs premiers, en général avec les images parentales) ;

- la voie politique. Il s'agit ici du rôle de la société qui agit de façon indirecte dans l'épuisement émotionnel du soignant. Nous avons vu les questions de reconnaissance de la charge psychique. Admettons maintenant que sans moyens économiques, il est difficile de produire un travail efficace. Mais le soutien de la « cité » passe surtout par le modèle politique. Avoir un leadership clair, un chef qui assume les décisions après les avoir requises démocratiquement auprès de son personnel. Permettre un sentiment d'appartenance pour augmenter la cohésion du groupe. Faciliter l'activité des membres de l'équipe en augmentant les interfaces entre eux. Admettre que les collaborateurs sont les meilleurs pour trouver les sources d'inefficacité. Réagir vite en cas extrême, comme avec les groupes de crise ou post-traumatiques qui permettront la reprise mentale après la sidération liée à l'imprévu. Ici, à l'hôpital, ces cas se produisent après un cumul de décès, la mort d'un patient particulièrement investi, une difficulté majeure de fonctionnement. Il est de nos jours étonnant de constater qu'autant de causes d'insatisfaction au travail se traduisent dans certains services par une réaction binaire : l'absentéisme. La compréhension des causes de l'absence est un premier pas face au burn-out. Elle se heurte bien souvent hélas au côté indémêlable des différentes composantes qui conduisent à la «maladie du travail ».

L'histoire de l'humanité montre que la dimension psychopathologique du travail est intrinsèque à la culture. Très souvent, l'acharnement au travail a produit les plus grandes œuvres. Mais même lorsque les humains ne savouraient pas, pendant leur existence, les effets de leur action, ils en recevaient les fruits dans un sentiment d'accomplissement et de sens donné à leur vie. Prendre soin d'autrui est une œuvre à sa manière. C'est une œuvre à plusieurs, sur le temps long de la vie. Elle ne doit pas pour autant donner l'impression d'une dilution dans le groupe et dans le temps. Pour que le travail soignant ne se résume pas au balancement entre épuisement et satisfaction, les approches institutionnelles, personnelles et politiques méritent des approfondissements aujourd'hui parfaitement repérés dans ce numéro. 